\title{
Solving a multi-objective vehicle scheduling- routing of interurban transportation fleet with the purpose of minimizing delays by Using the Differential Evolutionary Algorithm
}

\author{
Ali Javadi ${ }^{a^{*}}$, Mohammad Jafar Tarokh ${ }^{\mathrm{b}}$ and Shahnaz Piroozfar ${ }^{\mathrm{a}}$
}

${ }^{a}$ Department of Industrial Engineering, South Tehran Branch, Islamic Azad University, Tehran, Iran

${ }^{a}$ Department of Industrial Engineering, K.N. Toosi University of Technology, Tehran, Iran

\section{H R O N I C L E}

Article history:

Received December 10, 2013

Received in revised format

16 March 2014

Accepted May 102014

Available online

May 182014

Keywords:

Supply chain management

Vehicle routing problem

Interurban transportation

Multi-objective differential

evolutionary algorithm

\begin{abstract}
A B S T R A C T
Over the past three decades, the unified approach of the optimization of the logistic systems has become one of the most important aspects of optimizing the supply chain so that in recent decades it has had a large application in practice and has been used to increase the efficiency and effectiveness of the logistic fleet. The inter-urban transport networks by the terminals play an important role in logistic fleet and the goods distribution. In some cases, numbers of terminals face with an overload and others encounter with additional vehicles, which result delay in the load post and unnecessary car downtime. The present paper aims at modeling, scheduling and routing of the vehicles network and minimizing delays in order to create an optimal balance between the number of vehicles and the capacity of the car terminals to use the maximal capacity of vehicles. So that the multi-objective mathematical model is presented to quantify the regular transportation costs and to minimize the car downtime. The proposed model has two conflicting objectives where on tried to increase costs and the other decreases unused cars. Due to the high complexity of the problem, the multi-objective differential evolutionary algorithm (MODE) has been used. To prove the proposed algorithm, it has compared with the NSGA-II algorithm using four comparing indexes. The computational results show the superiority of the proposed algorithm.
\end{abstract}

\section{Introduction}

The shipping and transportation industry of each country represents the status of economy and technology development of that country. This industry could be stated as one of the most prominent factors in economic, cultural and social development of any community. There is also an increasing demand for transportation as a subsequent phase of the economic development and social welfare. Moreover, offering the transportation services with cheap prices has various gains for the development of any country. Consequently, we may expect an increase on the competition in the commercial environments. The products life cycle is getting shorter and the uncertainty in customers' demands has increased. In addition, the order performance time influences on the services and the variety of demands can be recognized as one of the important resources of uncertainty in supply

* Corresponding author. Tel: +989353714537

E-mail address: ali.javadi62@yahoo.com (A. Javadi)

(C) 2014 Growing Science Ltd. All rights reserved. doi: $10.5267 /$ j.uscm.2014.5.005 
Chain (Nozick \& Turnquist, 2001). Because of the increasing pressure for staying at the world market, the optimization of transportation networks throughout supply chain becomes an important challenge for the industries to reduce the costs and the delays and improves the services. The challenge requires an integrated supply chain management of optimizing scheduling problems in transportation networks.

\section{Literature}

The supply chain scheduling is relatively a new but important area of study and most portions of studies have happened during the last 10 years. In particular, the problem of the integrated production and distribution scheduling is one of the important studies after the work by Hall and Potts (2003). Chen (2010) recently, in an appropriate case study and is a good review of related studies. Two important components of a supply chain are production and distribution. Therefore, the coordination of production and delivery planning is one of the important issues of the supply chain scheduling. The classical problems of scheduling neglect the coordination with the transportation unit and the costs of delivery. However, in the recent years, classical scheduling problems have considered the cost of delivery in the supply chain scheduling approach. Hall and Potts (2005) studied the various problems concerning the scheduling, classification and delivery coordination and provided a method with the dynamic programming approach for minimizing the variety of target functions. One of the target functions that they studied was associated with minimizing the weighted sum of the delay time and the costs of transportation. Selvarajar and Zhang (2014) in their recent paper explored the problems associated with the supply chain scheduling in production, in order to reduce the costs and a depoestock deal. Today, the importance of delivery and the competitive environment intensifying for the services and products has devoted a special place for the supply chain management. There are various definitions proposed for the supply chain and almost all experts in defining the supply chain management, have emphasized the ultimate customer satisfaction as one of its main goals. One of the most important indicators for ultimate customer satisfaction is timely delivery of goods. The new research findings suggest that reducing delays in delivery of goods is one of the main concerns of the supply chain managers (Lockamy \& McCormack, 2004).

Generally, the vehicle routing problem is modeled on basis of the graph theory concepts. In this case, the graph nodes indicate customers and its edges indicate the available ways among customers. In accordance with each edge of the graph, the cost (time or distance) of travel is defined. Considering the ways that are one way or two ways and the costs of trips, the vector on non-vector graphs are defined. The vehicle routing problem plays an important role in the logistic and good distribution. The vehicle routing problem classical model is presented by a graph considering the limited capacity of vehicles in which its nodes are customers $(\mathrm{m})$ and its edges state the ways of communications among costumers. In this model, each costumer has the specified value of demand that all these demands (q) should be carried by a vehicle. Lack of access to the system in which each vehicle has the less free capacity may yields the loss of profit, padding the additional route by other vehicles, consumption of more fuel and the pollution of environment. It causes great losses to the environment and to the collection. There are extensive studies accomplished on the transportation scheduling (Berbeglia \& colleagues, 2007; Parragh et al., 2008; Parragh et al., 2008). In the vehicles scheduling field, Freling et al. (2001), for instance, considered one terminal and the purpose was vehicles scheduling for entering and exiting the terminal. Mingyong and Erbao (2010) considered improved differential evolutionary algorithm for solving vehicle routing problem with stimulus transportation and delivery to reduce costs and protect the environment. Neri and Tirronen (2010) considered the Differential Evolutionary (DE) and its recent progress toward removing its shortcomings. In another study, Huisman et al. (2004) studied the scheduling problem of vehicles with multiple terminals and tried to determine the optimum number of terminals. Zack (2009) designed a multi-criteria method for scheduling the public transportation vehicles in which terminals included the station stops and passengers are products. The aim is minimizing the passengers' wait time. In another work, "the 
multi-objective vehicle routing" provided by Banos et al. (2013) and the aim was to minimize the required distance to deliver goods and haul the workload imbalance using the meta-heuristic fusion algorithm by considering a time window. Erbao and Min Yang (2009) considered the vehicle routing problem with fuzzy demand (VRPFD) using the differential evolutionary algorithm. Borndorfer et al., (2004) and Mesquita et al. (2009), the vehicle routing scheduling and terminals crew were considered, simultaneously. In this paper, we aim to find the optimal route for each vehicle in the logistics to minimize the transportation cost and delays in fleet networks. The mathematical models are presented in the following assumptions. With respect to these assumptions, the double-objective mathematical model has offered.

\section{Model formulation}

In this section, first, the parameters associated with the scheduling and routing problem of vehicles in the fleet logistics are expressed, then the proposed model is provided.

\subsection{The basic assumptions of the problem}

The main assumptions of the problem are mentioned as follows,

- The model is a periodic planning. For each client (node), a set of meeting programs, which determine the access days, is prepared and among them only one program is selected for each node.

- If the client demand is more than the car capacity, it is likely to meet it more than one time in a day of planning. In the other words, there is a possibility to divide the services among cars.

- All customers can have the simultaneous demands both for the appeals and the delivery requirements.

- There is a time limitation for each car, which shows the maximum available time for each car to do services in each day of the planning period.

- The transportation fleet is composed of heterogeneous cars. These differences are the differentiation at car speed in travelling, the car capacity, the primary cost of using car in each day, the variable cost of using car at the distance unit and the maximum available time interval to use car.

- Cars are designed to get stuck in the target or go around city to get a stuck. In each case, the costs should be considered.

In this section, the problem is modeled in this study. First, all the used symbols are introduced. Then the mathematical model is presented. The Parameters include the followings:

\subsection{Parameters}

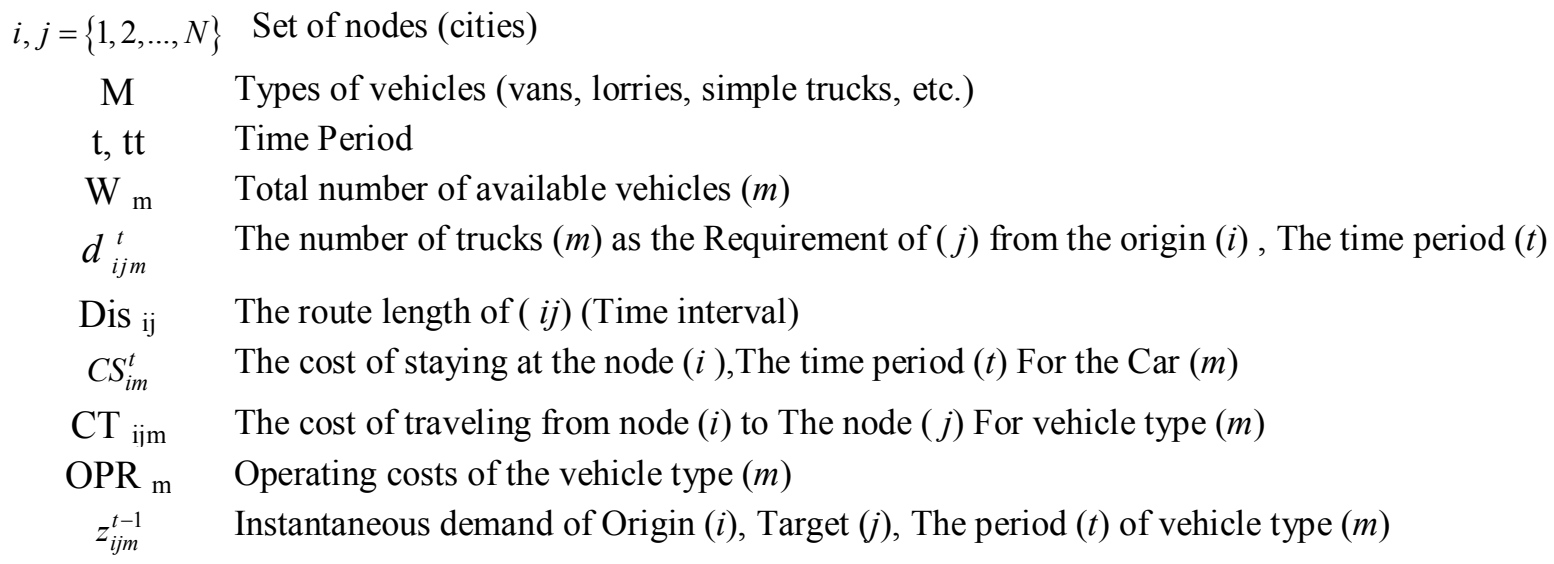


$\mathrm{Du}_{\mathrm{ij}} \quad$ Delivery time of target $(j)$ from origin $(i)$

\subsection{Variables}

A binary variable is considered, which is one if a car type $(m)$ is sent from the origin $(i)$ to the target (j) $($ Per $\mathrm{i} \neq \mathrm{j})$ in the time period $(t)$, and zero, otherwise.

A binary variable is also considered, which is one if a vehicle type $(m)$ is period $(t)$ is located in route (ij) and still has not reached its target, and zero, otherwise.

A binary variable is considered, which is one if a car type $(m)$ reaches from node $(i)$ to node $(j)$, and zero, otherwise.

$V_{i m}^{t}$ Total number of available vehicles in the origin $(i)$, car type $(m)$, the time period $(t)($ $V_{i m}$ is a determined quantity).

$F_{i m}^{t+}$ A positive value at time $(\mathrm{t})$, with the number of available vehicles at the origin $(i)$ and car type $(m)$

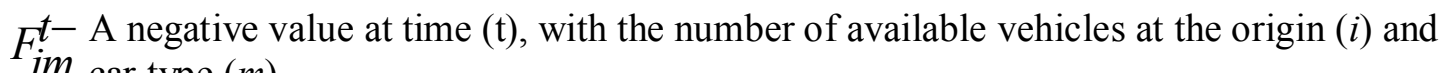

im car type $(m)$

$q_{i j}^{t}$ The number of empty vehicles dispatched from the origin $(i)$ to the target $(j)$, the time qijm period $(t)$ and car type $(\mathrm{m})$.

$\operatorname{Tar}_{i j}$ The delay of the delivery date from origin $(i)$ to the target $(j)$.

\subsection{Mathematical modeling of the problem}

$$
\begin{aligned}
& \min z_{1}=\sum_{t} \sum_{m} \sum_{i} \sum_{j} q_{i j m}^{t} \times C T_{i j m}+\sum_{t} \sum_{m} \sum_{i} C S_{i m}^{t} \times\left(V_{i m}-\sum_{j} q_{i j m}\right)+\sum_{m} O P R_{m} \\
& +\sum_{t} \sum_{i} \sum_{j} x_{i j m}^{t} \\
& \min _{2}=\sum_{j} \sum_{i} \operatorname{Tar}_{i j}
\end{aligned}
$$

subject to

$$
\begin{aligned}
& t+\text { Dis }_{i j} \\
& \sum_{j} \sum_{i} x_{i j m}^{t} \times d_{i j m}^{t}+\sum_{j} \sum_{i} y_{i j m}^{t} \times \sum_{t}^{i j} d_{i j m}^{t} \leq W_{m} ; \forall t \\
& \sum_{j} \sum_{i} x_{i j m}^{t} \times d_{i j m}^{t}+\sum_{j} y_{i j m}^{t} \times \sum_{t}^{t+D i s_{i j}} d_{i j m}^{t} \leq W_{i m} ; \forall t, i \\
& 1=\left[\left(\sum_{j} \sum_{i} x_{i j m}^{t-1} \times d{ }_{i j m}^{t-1}\right)+\left(\sum_{i} y_{i j m}^{t-1} \times \sum_{t}^{t+D i s_{i j}} d_{i j m}^{t-1}\right)+\sum_{j} z_{i j m}^{t-1}\right]+\sum_{j} s_{i j m}^{t} \times d_{i j m}^{t} ; \forall t, i, m \\
& x_{j i m}^{t-D i s_{i j}}=1 \text { then } s_{j i m}^{t}=1 ; \forall t, i, m
\end{aligned}
$$

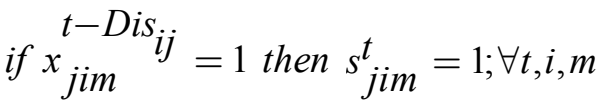

$$
\begin{aligned}
& \text { if } x_{j i m}^{t}=1 \text { then } y_{j i m}^{t t}=1 ; \forall i, m, t \leq t t \leq t+D i s_{j i}
\end{aligned}
$$




$$
\begin{aligned}
& \text { if } V_{i m}^{t}>0 \text { then } F_{i m}^{t+}=V_{i m}^{t} ; \forall i, m, t \\
& \text { if } V_{i m}^{t}<0 \text { then } F_{i m}^{t-}=V_{i m}^{t} ; \forall i, m, t \\
& F_{i m}^{t+} \times F_{i m}^{t-}=0 ; \forall i, m, t \\
& \text { if } V_{i m}^{t}<0 \& V_{j m}^{t}>0 \text { then } q_{j i m}^{t} \geq 0 ; \forall i, j, m, t \\
& \text { Tar }{ }_{i j}=\max \left\{\left[\sum_{m}\left(x_{i j m}^{t}\right) t-D i s_{i j}-D u e_{i j}\right], 0\right\} ; \forall i, j, t
\end{aligned}
$$

According to the symbols defined in the previous section and the assumptions briefly passed, the mathematical model of the problem in this section is presented. The objective function Eq. (1) maximizes the cost of staying in the garage, the cost of sending empty trucks to other cities as well as the operational costs of transportation. The objective function given in Eq. (2) minimizes the delay of delivery to all cities. In the constraint stated in Eq. (3), the total number of available vehicles in cities is less than the fleet number. In the constraint stated in Eq. (4), the total number of vehicles of each city can be sent to other locations and vehicles that have already been sent and still have not reached to their destination must be less than the total number of vehicles in the city. In the constraint Eq. (5), the number of vehicles in each node and in each limited period is calculated. According to Eq. (6), the vehicles that arrive to each node are calculated. By the constraint given in Eq. (7), the numbers of cars that have already been sent and are now traveling are calculated. The constraints (8) and (9) test which node requires additional vehicles and which node can send an empty truck to the other nodes. The constraint (10) states that at the same time one of the constraints (8) or (9) occurs. The number of vehicles that each node, which is faced with shortage can provide from other nodes is calculated by constraint (11). The constraint (12) indicates the amount of delay to satisfy every demand.

\section{Solution}

Since the proposed model is formulated as mixed integer programming and it is hard to solved the resulted problem for large-scale problems, it is necessary to use the meta-heuristic methods for solving it. The proposed multi-objective meta-heuristic evolutionary algorithm is used to solve the problem. The steps of the proposed algorithm are presented in more details.

\subsection{Primary answer display}

Displaying answer in the solution space and choosing its structure is effective on the efficiency and the answer quality. To start using algorithm, a set of initial solutions, considering the constraints of the problem are randomly produced.

\subsection{Differential Evolutionary Algorithm (DE)}

Considering the complexity of the problems and the importance of quick access to the answers and lack of appropriate accountability of the classical methods, nowadays the implementation of accidental search algorithms as a substitute for detailed problem search has been widely developed. One of these algorithms is Differential Evolutionary Algorithm (DE), which is a powerful method for optimization problems in the advanced search space. This algorithm was introduced by Storn and Price in 1995. This evolutionary algorithm begins its search process from a primary accidental population. Three factors including mutation, crossover and selection and three control parameters, 
namely population size NP, scale coefficient $\mathrm{F}$ and crossover possibility $\mathrm{CR}$ ) are used in the DE algorithm. The level process of this algorithm mentioned next.

\subsubsection{Initial population}

The primary population includes NP members that are randomly produced. Each of the answers should be at the range of answers. In a problem with $M$ dementia search space, the structure of $i^{\text {th }}$ member is $X^{i}=\left(x_{1}^{i}, x_{2}^{i}, \ldots, x_{m}^{i}\right)$.

\subsubsection{Mutations act}

For each member of $X^{i}$ at the population, the new answer at each repeat $t$ is produced according to Eq. (13).

$$
Y^{\dot{t}}(t)=X^{3}(t)+F \cdot\left(X^{\dot{1}}(t)-X^{2}(t)\right) \quad i=1,2, \ldots, N P
$$

where $r_{1}, r_{2}, r_{3} \in[1, \ldots, N P]$ are three unequal random integers. Scale coefficient $F$ is a fixed positive number that is often equals 5.0.

\subsubsection{Crossover act}

The new answer of $Z^{i}$ is produced by the combination of $X^{i}$ and $Y^{i}$ according to Eq. (14):

$z_{i}= \begin{cases}y_{j}(t) & \text { if rand } \leq C R \quad \text { or } \quad j=\text { jrand } \\ x_{j}(t) & \text { o.w. }\end{cases}$

where jrand $\in[1,2, \ldots, m]$

\subsubsection{The selection act}

If the amount of the new answer fitness is better than the previous solution, the new answer is substituted, otherwise, the previous answer remains in the repetition after search

$X^{i}(t+1)= \begin{cases}Z^{i} & \text { if } f i t\left(X^{i}(t)\right) \geq f i t\left(Z^{i}(t)\right) \\ X^{i} & \text { o.w. }\end{cases}$

where $f i t($.$) , shows the amount of answer fitness$

\subsubsection{Stop}

The search process is continued until the algorithm stop criterion becomes suitable. Usually the algorithm stop criterion could be selected on the basis of fixed amount of the fitness of the best answer changes or repeating algorithm to the specified amount. The main difference between the algorithm (DE) and the genetic algorithm (GA) is on the selection level. In GA, the chance of choosing one answer as one of the parents depends on the amount of its fitness, but in DE, all answers have equal chances. After the production of new answer using a self-adjusting mutation operator and 
a crossover operator, the new answer is compared with the previous answer and the new answer is substituted by the last one.

\section{Numerical results}

\subsection{Parameters setting}

It is well known that quality of an algorithm is influenced substantially by the values of its parameters. In this section, for optimizing behavior of the proposed algorithms, appropriate tuning of their parameters has been carried out. For this purpose, response surface methodology (RSM) is employed. RSM is defined as a collection of mathematical and statistical method-based experiments, which can be used to optimize processes. Regression equation analysis is used to evaluate the response surface model. First, those parameters, which could influence statistically on algorithm results are determined. Each factor is measured at two levels, which can be coded as -1 when the factor is at its low level $(L)$ and +1 when the factor is at its high level $(H)$. The coded variable can be defined as follows:

$$
X_{i}=\frac{r_{i}-\left(\frac{h+l}{2}\right)}{\left(\frac{h-l}{2}\right)}
$$

where $x_{i}$ and $r_{i}$ are coded as variable and real variable, respectively. $h$ and $l$ represent high level and low level of factor. After tuning the parameters, the set values have been tabulated in Table 1 .

Table 1

NS and MODE parameter settings

\begin{tabular}{ccccc}
\hline Algorithm & Parameters & Settings & Parameters & Settings \\
\hline \multirow{2}{*}{ MODE } & Population Size & 300 & $F$ & 0.8 \\
& Crossover Rate & 0.3 & NFC & 40000 \\
\hline
\end{tabular}

\section{NSGA-II assumptions and parameters value}

- The initial population is generated, randomly.

- Crossover operator is exerted on random selected solutions using one of these operators, namely one-point crossover, two-point crossover and uniform crossover, randomly.

- Mutation operator is executed on random selected solution using one of these operators, namely inversion, swap and reversion, randomly.

- The crossover and mutation ratios are set to 0.75 and 0.3 , respectively, using RSM method.

- Number of the initial population is set to 300 .

- The NFCs stopping criteria was set on 40000.

To compare the difference between performance of the differential evolutionary algorithm, all the input data were performed by both algorithms and compared by 4 indices of Quality ( Quality MetricQM ), Distance ( Spacing Metric-SM ), Diversity ( Diversity Metric-DM ) and the distance from the ideal point ( Mean Ideal Distance Metric-MID ). The numerical results are shown in Table 2 and 3. Table 2 is related to the problems with small size. The biggest problem is about the 35 cities, 8 transportation cars and 6 time periods. 
Table 2

The Comparison indices for problems with small size

\begin{tabular}{|c|c|c|c|c|}
\hline \multirow{2}{*}{$\begin{array}{c}\text { Problem No. } \\
i \times m \times t\end{array}$} & \multicolumn{2}{|c|}{ Quality Metric (QM) } & \multicolumn{2}{|c|}{ Spacing Metric (SM) } \\
\hline & NSGA-II & MODE & NSGA-II & MODE \\
\hline $20 \times 2 \times 2$ & 0.5 & 0.8 & 0.727 & 0.435 \\
\hline $20 \times 3 \times 4$ & 0.05 & 0.95 & 0.744 & 0.372 \\
\hline $20 \times 4 \times 6$ & 0 & 1 & 0.791 & 0.488 \\
\hline $30 \times 4 \times 2$ & 0 & 1 & 0.738 & 0.496 \\
\hline $30 \times 5 \times 4$ & 0 & 1 & 0.572 & 0.312 \\
\hline $30 \times 6 \times 6$ & 0.1 & 0.9 & 0.499 & 0.574 \\
\hline $35 \times 6 \times 4$ & 0.25 & 0.75 & 0.793 & 0.645 \\
\hline $35 \times 8 \times 6$ & 0 & 1 & 0.983 & 0.548 \\
\hline Problem No. & \multicolumn{2}{|c|}{ Diversity Metric (DM) } & \multicolumn{2}{|c|}{ Mean Ideal Distance (MID) } \\
\hline $\mathrm{i} \times \mathrm{m} \times \mathrm{t}$ & NSGA-II & MODE & NSGA-II & MODE \\
\hline $20 \times 2 \times 2$ & 0.894 & 1.087 & 0.716 & 0.657 \\
\hline $20 \times 3 \times 4$ & 0.831 & 0.903 & 0.548 & 0.437 \\
\hline $20 \times 4 \times 6$ & 0.975 & 1.319 & 0.756 & 0.518 \\
\hline $30 \times 4 \times 2$ & 1.076 & 1.188 & 0.849 & 0.623 \\
\hline $30 \times 5 \times 4$ & 1.354 & 1.456 & 0.684 & 0.258 \\
\hline $30 \times 6 \times 6$ & 0.994 & 1.288 & 0.869 & 0.710 \\
\hline $35 \times 6 \times 4$ & 1.148 & 1.329 & 0.776 & 0.534 \\
\hline $35 \times 8 \times 6$ & 0.537 & 1.463 & 0.476 & 0.264 \\
\hline
\end{tabular}

From the results, it is observed that the proposed algorithm is more efficient than GA in all indices. The quality index, which is the most important one, has a value close to 1 for the proposed algorithm, indicating the above algorithm can find near-optimal Pareto solutions. Because the distance index shows the degree of distribution of Pareto solutions, so whatever this amount be less, the algorithm has a better function and results that Pareto solutions are equally distributed in the space. The diversity index shows the wide variety of searched Pareto by each algorithm. Whatever the amount be higher, shows the capabilities of the algorithm in the search space. Finally, whatever the distance index be less than the ideal point, represents the Pareto is closer to the ideal solution than can be observed in both tables.

Table 3

The Comparison Indices for problems with large size

\begin{tabular}{|c|c|c|c|c|}
\hline \multirow{2}{*}{$\begin{array}{l}\text { Problem No. } \\
i \times \mathrm{m} \times \mathrm{t}\end{array}$} & \multicolumn{2}{|c|}{ Quality Metric (QM) } & \multicolumn{2}{|c|}{ Spacing Metric (SM) } \\
\hline & NSGA-II & MODE & NSGA-II & MODE \\
\hline $50 \times 8 \times 6$ & 0 & 1 & 1.042 & 0.771 \\
\hline $50 \times 10 \times 12$ & 0 & 1 & 0.709 & 0.593 \\
\hline $70 \times 8 \times 12$ & 0 & 1 & 1.072 & 0.622 \\
\hline $70 \times 10 \times 14$ & 0.05 & 0.95 & 0.653 & 0.401 \\
\hline $100 \times 10 \times 12$ & 0 & 1 & 1.036 & 0.392 \\
\hline $100 \times 12 \times 16$ & 0 & 1 & 0.920 & 0.301 \\
\hline $150 \times 14 \times 18$ & 0 & 1 & 0.731 & 0.423 \\
\hline $200 \times 18 \times 24$ & 0 & 1 & 1.138 & 0.558 \\
\hline Problem No. & \multicolumn{2}{|c|}{ Diversity Metric (DM) } & \multicolumn{2}{|c|}{ Mean Ideal Distance (MID) } \\
\hline $\mathrm{i} \times \mathrm{m} \times \mathrm{t}$ & NSGA-II & MODE & NSGA-II & MODE \\
\hline $50 \times 8 \times 6$ & 1.042 & 1.142 & 0.684 & 0.453 \\
\hline $50 \times 10 \times 12$ & 1.044 & 1.172 & 0.983 & 0.774 \\
\hline $70 \times 8 \times 12$ & 0.871 & 1.249 & 0.769 & 0.590 \\
\hline $70 \times 10 \times 14$ & 0.430 & 1.020 & 0.982 & 0.439 \\
\hline $100 \times 10 \times 12$ & 1.132 & 1.549 & 0.684 & 0.208 \\
\hline $100 \times 12 \times 16$ & 0.594 & 0.649 & 0.659 & 0.364 \\
\hline $150 \times 14 \times 18$ & 0.983 & 0.993 & 0.830 & 0.601 \\
\hline $200 \times 18 \times 24$ & 0.392 & 0.670 & 0.755 & 0.554 \\
\hline
\end{tabular}


In order to show the performance of the algorithm, comparing indices for different problems are shown schematically in Fig. 1 to Fig. 4. In all figures, it is clear that the proposed algorithm has a significant difference with the genetic algorithm.

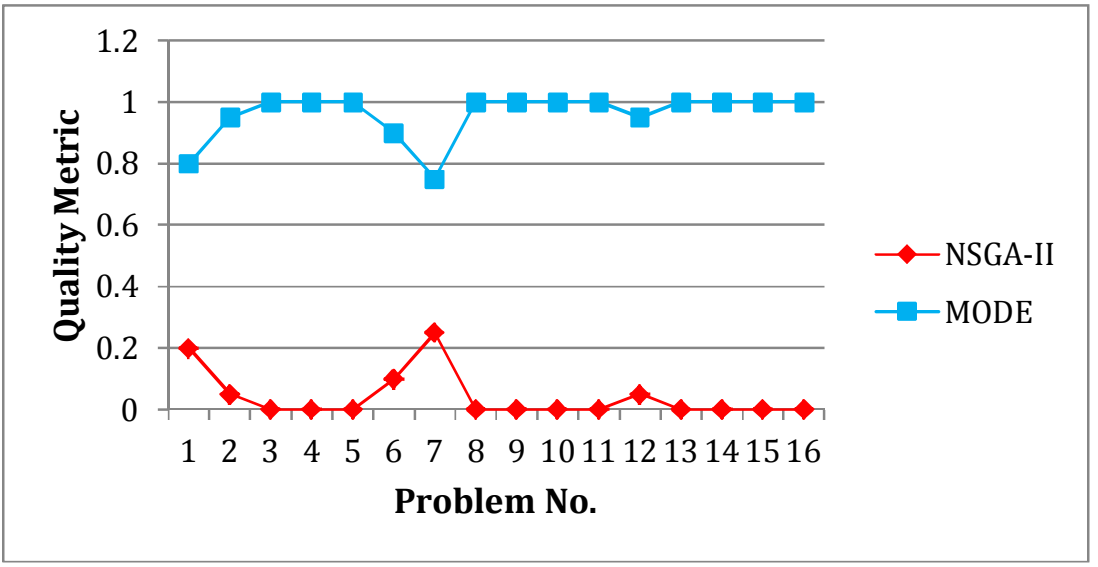

Fig. 1. The Comparison of the Quality Index

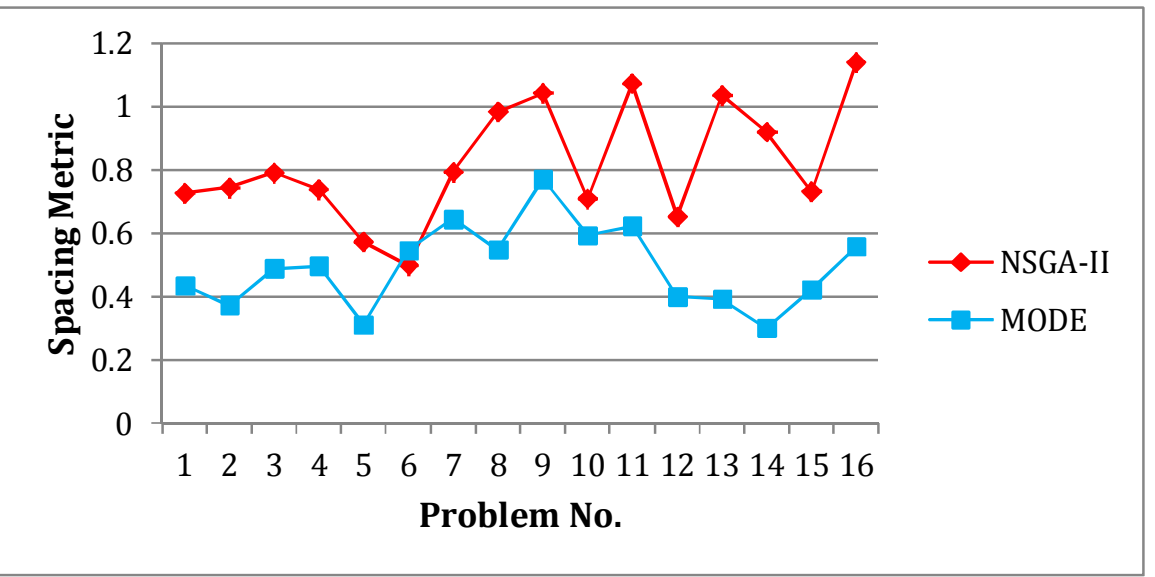

Fig. 2. The comparison of the distance index

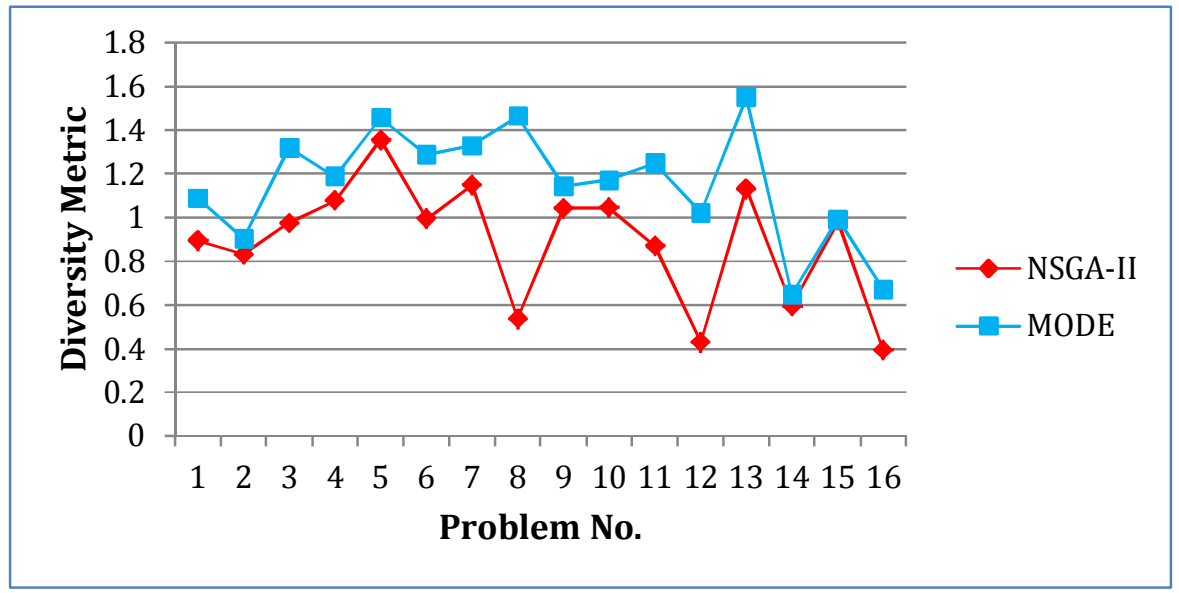

Fig. 3. The Comparison of the variety index 


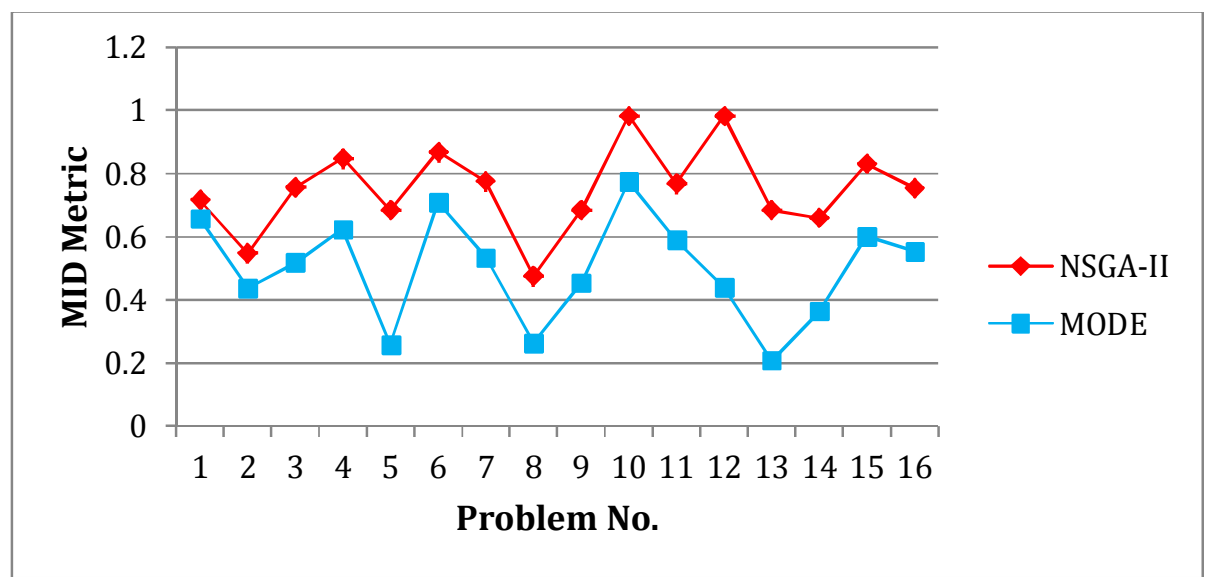

Fig. 4. The Comparison of the distance from the ideal point index

In order to perform a comparison between two algorithms, the paired t-test is used and significant difference between the algorithms is proved.

Table 4

The Paired t-test to compare the two algorithms

\begin{tabular}{|c|c|c|c|c|c|c|c|c|c|}
\hline \multirow{3}{*}{ Metric } & \multirow{3}{*}{ Pair } & \multicolumn{5}{|c|}{ Paired Differences } & \multirow{3}{*}{$t$} & \multirow{3}{*}{$d f$} & \multirow{3}{*}{$\begin{array}{c}\text { Sig. } \\
(2- \\
\text { tailed) }\end{array}$} \\
\hline & & \multirow{2}{*}{ Mean } & \multirow{2}{*}{$\begin{array}{c}\text { Std. } \\
\text { Deviation }\end{array}$} & \multirow{2}{*}{$\begin{array}{l}\text { Std. } \\
\text { Error } \\
\text { Mean }\end{array}$} & \multicolumn{2}{|c|}{$\begin{array}{l}95 \% \text { CI of the } \\
\text { Difference }\end{array}$} & & & \\
\hline & & & & & Lower & Upper & & & \\
\hline Quality & \multirow{4}{*}{ MODE-NSGA-II } & .73 & .33 & .041 & .64 & .81 & 17.6 & 15 & .00 \\
\hline SM & & -.25 & .20 & .025 & -.30 & -.20 & -10.37 & 15 & .00 \\
\hline $\mathrm{DM}$ & & .21 & .32 & .039 & .13 & .29 & 5.50 & 15 & .00 \\
\hline MID & & -.29 & .23 & .028 & -.35 & -.23 & -10.41 & 15 & .00 \\
\hline
\end{tabular}

Given the significant levels of paired t-test and the fact that all values are less than 0.05 , according to NSGA-II we can conclude that the proposed algorithm is significantly different in all four indices. So the proposed algorithm can be used with high confidence in other areas.

\section{Conclusions}

The transportation companies mainly face with a problem called the existence of one way-load demands. It means that most cars are dispatched for a mission but when in their return, they're forced to come back without any load. This is a typical kind of wasting at the transportation firms. On the other hand, the unnecessary car downtime is another aspect of wasting. Therefore, it is desired for al firms that the dispatched cars have the load demands without any car downtime. On the other side, in today world, many try to optimize problems in terms of various criteria, which are often in conflict with each other. In most organizations, decision makers prefer to have different options in order to select the optimum option for the conditions so they cannot certainly consider one option better than another. Thus, considering different objective functions simultaneously, diverse solutions are provided for the decision makers to enable them choose the best option by comparing the current conditions and various aspects. 
The present paper has tried to model, schedule and route vehicles network by minimizing delays in order to create an optimal balance between the number of vehicles and the capacity of the car terminals to use the maximal capacity of vehicles. The goal was to present the multi-objective mathematical model to quantify the regular transportation costs and to minimize the car downtime. The proposed model has two conflicting objectives where one tried to increase costs and the other tried to decrease cars sleep. Due to the high complexity of the problem, the multi-objective differential evolutionary algorithm (MODE) has been used. To prove the proposed algorithm, the problems was examined for two sizes; the big one and the small one and the given Pareto answers were compared with the NSGA-II algorithm using four comparing indices; the distance from the ideal point, quality, distance and variety. The computational results show the superiority of the proposed algorithm. Continuing to raise the validity of the results, the paired t-test was used which resulted the significant differences between the two comparing algorithms. The use of other algorithms and also applying new hypotheses to this problem can be proposed the future researchers.

\section{References}

Banos, R., Ortega, J., Gil, C., Marquez, A. L., \& De Toro, F. (2013). A hybrid meta-heuristic for multi-objective vehicle routing problems with time windows.Computers \& Industrial Engineering, 65(2), 286-296.

Berbeglia, G., Cordeau, J. F., Gribkovskaia, I., \& Laporte, G. (2007). Static pickup and delivery problems: a classification scheme and survey. Top, 15(1), 1-31.

Borndorfer, R., Lobel, A \& Weider, S. (2004). A Bundle Method for Integrated Multi-Depot Vehicle and Duty Scheduling in Public Transit. Computer-Aided Systems in Public Transport (M. Hickman, P. Mirchandani, S. Voss, eds). Lecture notes in economics and mathematical systems, 600, 3-24.

Chen, Z. L. (2010). Integrated Production and Outbound Distribution Scheduling: Review and Extensions. Operations Research, 58 (1), 130-148.

Erbao, C., \& Mingyong, L. (2009). A hybrid differential evolution algorithm to vehicle routing problem with fuzzy demands. Journal of computational and applied mathematics, 231(1), 302310.

Freling, R., Wagelmans, A. P., \& Paixão, J. M. P. (2001). Models and algorithms for single-depot vehicle scheduling. Transportation Science, 35(2), 165-180.

Hall, N. G., \& Potts, C. N. (2003). Supply chain scheduling: Batching and delivery. Operations Research, 51(4), 566-584.

Hall, N. G., \& Potts, C. N. (2005). The coordination of scheduling and batch deliveries. Annals of Operations Research, 135(1), 41-64.

Huisman, D., Freling, R., \& Wagelmans, A. P. (2004). A robust solution approach to the dynamic vehicle scheduling problem. Transportation Science,38(4), 447-458.

Lockamy III, A., \& McCormack, K. (2004). Linking SCOR planning practices to supply chain performance: An exploratory study. International Journal of Operations \& Production Management, 24(12), 1192-1218.

Mesquita, M., Paias, A \& Respicio, A.(2009). Branching Approaches for Integrated Vehicle and Crew Scheduling. Public Transport Planning and Operations 1(1), 21-37,.

Mingyong, L., \& Erbao, C. (2010). An improved differential evolution algorithm for vehicle routing problem with simultaneous pickups and deliveries and time windows. Engineering Applications of Artificial Intelligence, 23(2), 188-195.

Neri, F., \& Tirronen, V. (2010). Recent advances in differential evolution: a survey and experimental analysis. Artificial Intelligence Review, 33(1-2), 61-106.

Nozick, L. K., \& Turnquist, M. A. (2001). Inventory, transportation, service quality and the location of distribution centers. European Journal of Operational Research, 129(2), 362-371. 
Parragh, S. N., Doerner, K. F., \& Hartl, R. F. (2008). A survey on pickup and delivery problems. Journal für Betriebswirtschaft, 58(1), 21-51.

Parragh, S. N., Doerner, K. F., \& Hartl, R. F. (2008). A survey on pickup and delivery problems. Journal für Betriebswirtschaft, 58(1), 21-51.

Selvarajah, E., \& Zhang, R. (2014). Supply chain scheduling at the manufacturer to minimize inventory holding and delivery costs. International Journal of Production Economics, 147, $117-$ 124.

Zak, J., Jaszkiewicz, A., \& Redmer, A. (2009). Multiple criteria optimization method for the vehicle assignment problem in a bus transportation company. Journal of Advanced Transportation, 43(2), 203-243. 